\title{
IgG4-Related Hypertrophic Pachymeningitis with Skull Base Involvement Presenting with Isolated Glossopharyngeal and Vagus Nerve Palsy: A Case Report
}

\author{
Risa Suzuki ${ }^{1}$, Reiji Koide ${ }^{1}$, Shuya Hirano ${ }^{1}$, Takafumi Mashiko ${ }^{1}$, Tadashi Ozawa ${ }^{1}$, \\ Kumiko Miura ${ }^{1}$, Kosuke Matsuzono ${ }^{1}$, Saeko Uemura ${ }^{2}$, Ryota Tanaka ${ }^{1}$, Harushi Mori ${ }^{3}$ and \\ Shigeru Fujimoto ${ }^{1}$
}

\begin{abstract}
:
We herein report a 70-year-old man diagnosed with IgG4-related hypertrophic pachymeningitis with skull base involvement, who presented with isolated glossopharyngeal and vagus nerve palsy. Contrast-enhanced magnetic resonance imaging (MRI) showed enhanced dural thickening of the posterior clivus and skull base involvement. When a patient with hypertrophic pachymeningitis presents with isolated cranial neuropathy without systemic manifestations or definite MRI abnormalities, it is difficult to make a diagnosis, and the patient may be misdiagnosed. This case suggests that a detailed radiological evaluation including contrast enhancement of the skull base is very important in patients with isolated glossopharyngeal and vagus nerve palsy.
\end{abstract}

Key words: IgG4-related disease, hypertrophic pachymeningitis, glossopharyngeal and vagus nerve palsy

(Intern Med Advance Publication)

(DOI: 10.2169/internalmedicine.8144-21)

\section{Introduction}

Hypertrophic pachymeningitis (HP) is an inflammatory condition that causes a diffuse or localized thickening of the dura mater. Although headache and cranial neuropathies are the most common neurological manifestations of HP, when a patient presents with isolated cranial neuropathy without systemic manifestations or definite magnetic resonance imaging (MRI) abnormalities, it is difficult to make a diagnosis, and the patient may be misdiagnosed.

We herein report a 70-year-old man diagnosed with IgG4related HP with skull base involvement, who presented with glossopharyngeal and vagus nerve palsy. MRI without contrast medium failed to reveal any abnormalities; however, contrast-enhanced MRI showed enhanced dural thickening of the posterior clivus and the cerebellar tentorium. This case suggests that a detailed radiological evaluation includ- ing contrast enhancement of the skull base is very important in patients with isolated glossopharyngeal and vagus nerve palsy, and an early and appropriate diagnosis and treatment of HP are indispensable for reducing neurological sequelae.

\section{Case Report}

A 70-year-old previously healthy Japanese man was admitted to our hospital in November 2019 owing to gradually worsening hoarseness of voice and swallowing difficulty. On admission, neurological examinations revealed glossopharyngeal and vagus nerve palsy (severe hoarseness of voice, swallowing difficulty, soft palate palsy, and poor gag reflex). Other cranial nerve functions showed no abnormalities, and no motor or sensory deficits were observed. The laryngoscopic findings were bilateral vocal fold immobility and fixation in the paramedian position. Routine laboratory examinations revealed no remarkable findings except for an

${ }^{1}$ Division of Neurology, Department of Medicine, Jichi Medical University School of Medicine, Japan, ${ }^{2}$ Department of Otorhinolaryngology, Jichi Medical University School of Medicine, Japan and ${ }^{3}$ Department of Radiology, Jichi Medical University School of Medicine, Japan Received: June 15, 2021; Accepted: October 3, 2021; Advance Publication by J-STAGE: November 20, 2021 Correspondence to Dr. Reiji Koide, reiji_koide@jichi.ac.jp 

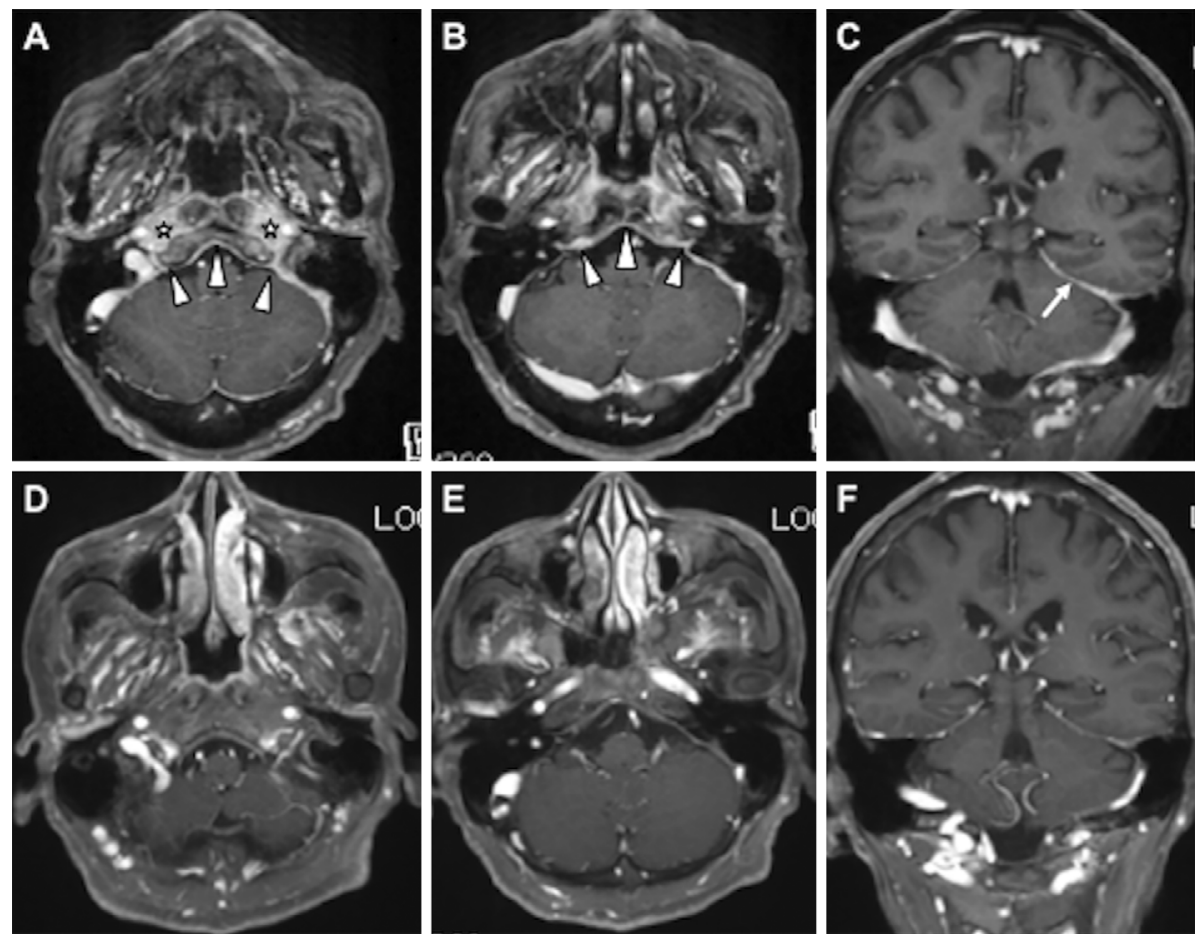

Figure. Brain MRI findings on admission (A-C) and after treatment (D-F). Axial (A, B, D, E) and coronal (C, F) gadolinium-enhanced images. On admission (obtained in December 2019), contrastenhanced MR images showed dural thickening involving the posterior clivus (arrowheads), surrounding the jugular foramen (arrowheads) and the left cerebellar tentorium (arrow). In addition, infiltrative lesions involving the clivus, petrous apex, and prevertebral and carotid spaces were observed (white stars). After treatment (obtained in March 2020), a reduction in dural thickening and enhancement as well as improvement of the skull base infiltration were observed.

elevated erythrocyte sedimentation rate $(44 \mathrm{~mm} / \mathrm{h})$. An immunologic analysis revealed an elevated serum $\mathrm{IgG} 4$ level (136 $\mathrm{mg} / \mathrm{dL}$; normal range, 4.5-117 $\mathrm{mg} / \mathrm{dL}$ ). Serum angiotensin-converting enzyme levels were normal. Tests for antinuclear antibodies, rheumatoid factor, anti-SS-A/SS-B antibodies, myeloperoxidase- and proteinase 3-antineutrophil cytoplasmic antibodies (MPO-, and PR3-ANCA), antiacetylcholine receptor, and anti-muscle-specific tyrosinase antibodies were all negative. Cerebrospinal fluid (CSF) studies showed no remarkable findings. CSF cultures for bacteria and fungi were negative, and polymerase chain reaction for Mycobacterium tuberculosis was also negative. MRI without contrast medium could not reveal any abnormalities; however, contrast-enhanced MRI showed enhanced dural thickening of the posterior clivus and the cerebellar tentorium. In addition, infiltrative lesions in the clivus, petrous apex, and the prevertebral and carotid spaces were observed (FigureA-C). Based on these findings, he was diagnosed with IgG4-related HP with skull base involvement presenting with glossopharyngeal and vagus nerve palsy.

He started treatment with the oral administration of prednisolone at $40 \mathrm{mg} / \mathrm{day}$, which was then slowly tapered. At two months after the start of treatment, soft palate palsy and dysphagia gradually began to improve. At this point, IgG4 levels had already normalized, and follow-up MRI showed a reduction in dural thickening and enhancement (FigureD-F).
His voice hoarseness took a long time to improve and gradually improved about six months after treatment. In May 2021, he was in stable remission with oral administration of corticosteroids at $5 \mathrm{mg} / \mathrm{day}$, and MRI showed no recurrence of HP.

\section{Discussion}

IgG4-related disease (IgG4-RD) is an immune-mediated multisystem disorder characterized by tissue infiltration with lymphocytes and IgG4-secreting plasma cells. Common clinical presentations include autoimmune pancreatitis, salivary and lacrimal gland enlargement, orbital inflammation, and retroperitoneal fibrosis (1). Central and peripheral nervous system complications of IgG4-RD are relatively uncommon, but HP, hypophysitis, and neuropathy have been reported (2-4). Skull base and sinonasal involvement is also rare, although there have been some reports $(5,6)$. The present case had no lesions on which a biopsy could be performed. Therefore, this case is classified as "possible" based on the diagnostic criteria for IgG4-RD (7).

$\mathrm{HP}$ is an inflammatory condition that causes a diffuse or localized thickening of the dura mater. It is associated with tuberculosis, fungal meningitis, syphilis, rheumatoid arthritis, granulomatosis with polyangiitis, sarcoidosis, and IgG4RD (2). The clinical manifestations of HP vary and depend 
on the location of lesions. The most common symptoms are headache and cranial nerve palsy. Cranial nerve VIII is the most frequently affected nerve, followed by cranial nerves II, V, VII, IX, X, and XII. Isolated glossopharyngeal and vagus nerve palsy is a rare condition in HP (8). It is interesting that cranial nerve palsy can cause lower cranial nerve palsies due to HP, although IgG4-RD is known to directly affect the trigeminal nerves (9).

Treatment for HP varies based on the underlying mechanism. Corticosteroids is conventionally recommended as the first-line therapy, and for refractory cases we consider additional immunosuppressive therapies. Early therapy using steroids and immunosuppressants can prevent neurologic complications (10).

The diagnosis of IgG4-related HP is often difficult, particularly in patients who develop isolated cranial neuropathy without systemic manifestations or definite MRI abnormalities. In patients with isolated glossopharyngeal and vagus nerve palsy, as seen in the present case, physicians should be careful not to overlook subtle changes in the skull base on MR images including gadolinium enhancement and should thoroughly rule out a treatable immunological disorder including localized HP.

The authors state that they have no Conflict of Interest (COI).

\section{References}

1. Wallace ZS, Deshpande V, Mattoo H, Mahajan VS, Kulikova M, Pillai S, Stone JH. IgG4-related disease: clinical and laboratory features in one hundred twenty-five patients. Arthritis Rheumatol
67: 2466-2475, 2015.

2. Wallace ZS, Carruthers MN, Khosroshahi A, et al. IgG4-related disease and hypertrophic pachymeningitis. Medicine 92: 206-216, 2013.

3. Chan SK, Cheuk W, Chan KT, Chan JKC. IgG4-related sclerosing pachymeningits: a previously unrecognized form of central nervous system involvement in IgG4-related sclerosing disease. Am J Surg Pathol 33: 1249-1252, 2009.

4. Ohyama K, Koike H, Iijima M, et al. IgG4-related neuropathy: a case report. JAMA Neurol 70: 502-505, 2013.

5. Ryu G, Cho HJ, Lee KE, Lee JJ, Hong SD, Kim HY, Chung SK, Dhong HJ. Clinical significance of $\mathrm{IgG} 4$ in sinonasal and skull base inflammatory peseudotumor. Eur Arch Otorhinolaryngol 276: 2465-2473, 2019.

6. Detiger SE, Karim F, Monserez D, Verdijk R, van Hagen M, Paridaens D, van Laar J. IgG4-related disease of skull base: case series of 3 patients with headache. World Neurosurg 134: 536-539, 2020.

7. Umehara H, Okazaki K, Masaki Y, et al. Comprehensive diagnostic criteria for IgG4-related disease (IgG4-RD), 2011. Mod Rheumatol 22: 21-30, 2012.

8. Kosumi Y, Nakamura H, Watanabe R, Watanabe T, Horita T. Glossopharyngeal/vagus nerve disorder due to IgG4-related hypertrophic pahymeningitis. Rheumatology 58: 1513, 2019.

9. Wu PC, Tien PT, Li YH, Chen RY, Cho DY. IgG4-related cerebral pseudotumor with perineural spreading along branches of the trigeminal nerves causing compressive optic neuropathy: a case report. Medicine 96: e8709, 2017.

10. Levraut M, Cohen M, Bresch S, et al. Immunoglobulin G4-related hypertrophic pachymeningitis: A case-oriented review. Neurol Neuroimmunol Neuroinflamm 6: e568, 2019.

The Internal Medicine is an Open Access journal distributed under the Creative Commons Attribution-NonCommercial-NoDerivatives 4.0 International License. To view the details of this license, please visit (https://creativecommons.org/licenses/ by-nc-nd/4.0/).

(C) The Japanese Society of Internal Medicine Intern Med Advance Publication 